\title{
PIK3CA mutations in the kinase domain (exon 20) of uterine endometrial adenocarcinomas are associated with adverse prognostic parameters
}

\author{
Lluis Catasus, Alberto Gallardo, Miriam Cuatrecasas and Jaime Prat \\ Department of Pathology, Hospital de la Santa Creu i Sant Pau, Autonomous University of Barcelona, \\ Barcelona, Spain
}

\begin{abstract}
Mutations of the oncogene PIK3CA occur frequently in endometrial carcinomas, but their prognostic significance is unclear. To determine the clinicopathological and molecular implications of these mutations, PIK3CA status was investigated in 109 endometrial (102 endometrioid and 7 mixed) carcinomas and the results were compared with clinicopathological parameters associated with prognosis. Tumors were also investigated for microsatellite instability and PTEN, $\beta$-catenin gene (CTNNB1), $K-R A S$, and $B-R A F$ mutations. We found 35 PIK3CA somatic missense mutations in $32(29 \%)$ endometrial carcinomas. Eighteen mutations occurred in exon 20 (kinase domain), and 17 in exon 9 (helical domain). Almost all mutated tumors were pure endometrioid adenocarcinomas. All tumors with PIK3CA mutations exhibited myometrial invasion $(P=0.032)$. Lymphovascular invasion was found more frequently in mutated $(28 \%)$ than nonmutated carcinomas (18\%). Histological grade varied significantly according to the location of the PIK3CA mutations whether in exon 9 or exon 20 $(P=0.033)$. The frequency of exon 9 mutations was higher in grade 1 carcinomas $(57 \%)$ than in grade $2(29 \%)$ or grade $3(14 \%)$ tumors. Conversely, mutations in exon 20 were more common in grade $3(60 \%)$ than in grade 2 $(20 \%)$ or grade $1(20 \%)$ carcinomas. None of the tumors confined to the endometrium (stage IA) had PIK 3 CA mutations. Furthermore, whereas $64 \%$ of adenocarcinomas with exon 9 mutations had invaded $\leq 1 / 2$ of the myometrial thickness (stage IB), 73\% of tumors with exon 20 mutations had either deeper myometrial invasion (stage IC) or cervical involvement (stage II) $(P=0.045)$. PIK3CA mutations coexisted with microsatellite instability and mutations in PTEN, CTNNB1, K-RAS, and B-RAF genes. These results favor that PIK3CA mutations are associated with myometrial invasion and, moreover, that tumors harboring PIK3CA mutations in exon $\mathbf{2 0}$ are frequently high-grade, deeply invasive endometrial carcinomas that tend to exhibit lymphovascular invasion.
\end{abstract}

Modern Pathology (2008) 21, 131-139; doi:10.1038/modpathol.3800992; published online 14 December 2007

Keywords: endometrial carcinoma; histological grade; myometrial invasion; PIK3CA

Endometrial carcinoma is the most common malignant tumor of the female genital tract in the Western world. ${ }^{1}$ Over $80 \%$ of cases are estrogen-related well-differentiated to moderately well-differentiated endometrioid adenocarcinomas, which are confined to the uterine corpus at diagnosis (stage I), and thus most of them can be cured. While favorable in most cases, the prognosis of stage I endometrioid adenocarcinomas varies significantly depending on

Correspondence: Professor J Prat, MD, FRCPath, Department of Pathology, Hospital de la Santa Creu i Sant Pau, Avda. Sant Antoni M Claret 167, Barcelona 08025, Spain.

E-mail: jprat@santpau.es

Received 10 September 2007; revised 09 November 2007; accepted 15 November 2007; published online 14 December 2007 histological grade, depth of myometrial invasion, and presence or absence of lymphovascular invasion. Patients with high-grade tumors that invade $>1 / 2$ of the myometrial thickness or exhibit lymphovascular invasion have a high risk of regional lymph node metastases and require adjuvant therapy. ${ }^{2}$

Within the last decade, several genetic alterations, including microsatellite instability, ${ }^{3-5}$ PTEN alterations, ${ }^{6-8}$ and mutations of $K-R A S^{9-11}$ and CTNNB1 $(\beta$-catenin $),{ }^{12,13}$ have been discovered in endometrioid adenocarcinomas and their prognostic significance widely investigated. Although microsatellite instability was initially thought to be associated with a favorable outcome, ${ }^{14}$ recent data, obtained after excluding nonendometrioid carcinomas, have 
shown that the frequency of microsatellite instability is higher in high-grade and advanced stage tumors with deep myometrial and lymphovascular invasion. ${ }^{15,16}$ Similarly, earlier investigations suggested that PTEN mutations were associated with low-stage, nonmetastatic disease, and prolonged survival; ${ }^{17}$ however, subsequent studies reported that mutations only outside exons 5-7 of PTEN might represent a molecular predictor of favorable survival, independent of the clinical and pathological characteristics of the tumors. ${ }^{18}$ Also, promoter methylation of PTEN has been associated with advanced stage in endometrial carcinoma. ${ }^{19}$ In contrast, CTNNB1 ( $\beta$-catenin) mutations are thought to represent a separate pathway in estrogen-driven endometrial carcinogenesis, resulting in low-grade, low-stage endometrioid adenocarcinomas with squamous differentiation and excellent prognosis. ${ }^{20,21}$

The phosphatidylinositol 3'-kinase (PI3K)/AKT signaling pathway is frequently activated in multiple human epithelial cancers, including endometrioid adenocarcinomas. ${ }^{22-25}$ In response to cell-surface receptor signals, PI3K phosphorylates phosphatidylinositol 4,5-biphosphate to generate the second messenger phosphatidylinositol 3,4,5triphosphate. This, in turn, activates the AKT oncogenic pathway, directly counteracting the actions of the lipid phosphatase PTEN. ${ }^{26}$ Activated AKT regulates the expression of various downstream target genes that inhibit apoptosis and promote cell proliferation. A high frequency of mutations of the oncogene PIK3CA, which encodes the p110 $\alpha$ catalytic subunit of PI3K, has been recently identified in endometrioid adenocarcinomas. ${ }^{25,27,28}$ These mutations are usually of the missense type and clustered in exon 9 (residues E542 and E545 of the helical domain) and exon 20 (residue H1047 of the kinase domain). However, in contrast to other epithelial cancers, no association has been found between the distribution of PIK3CA mutations and the traditional prognostic parameters of endometrioid adenocarcinomas. ${ }^{25,28}$ Given the reported role of PIK3CA mutations in tumor invasion and metastases, ${ }^{29}$ we were interested in determining the clinicopathological impact of PIK3CA mutations in a large series of endometrial adenocarcinomas and their relationship with other genetic alterations also common in these tumors. We found that all carcinomas with PIK3CA mutations exhibited myometrial invasion and, furthermore, histological grade and depth of myometrial invasion varied significantly according to the distribution of mutations between exons 9 and 20 .

\section{Materials and methods}

\section{Tumor Samples and Genomic DNA}

One hundred and nine unselected endometrial adenocarcinomas were retrieved from the Tumor
Bank of the Department of Pathology, Hospital de la Santa Creu i Sant Pau, Autonomous University, Barcelona, Spain. All available hysterectomy slides were reviewed using the World Health Organization classification, ${ }^{30}$ and clinical data were obtained from the medical record. Tumors were staged and graded according to the International Federation of Gynaecology and Obstetrics (FIGO) guidelines. Genomic DNA was extracted by a standard procedure from frozen tumor and corresponding normal tissues. All cases were anonymized, and the study was approved by the institutional ethics committee.

\section{PIK3CA, PTEN, $\beta$-Catenin Gene, K-RAS, and B-RAF Mutational Analysis}

PIK3CA, PTEN, $\beta$-catenin gene (CTNNB1), K-RAS, and $B-R A F$ mutations were assessed on tumor DNA by polymerase chain reaction (PCR) amplification and subsequent sequencing analysis. Mutational analysis was performed using previously reported PCR conditions and primers for exons 9 and 20 of PIK3CA, ${ }^{31}$ exons $1-9$ of PTEN, ${ }^{8}$ exon 3 of CTNNB1, ${ }^{13}$ exon 1 of $K-R A S,{ }^{11}$ and exons 11 and 15 of $B-R A F$ genes. ${ }^{32}$ The thermal cycling conditions included an initial $12 \mathrm{~min}$ at $94^{\circ} \mathrm{C}$, followed by 40 cycles of $45 \mathrm{~s}$ at $94^{\circ} \mathrm{C}, 45 \mathrm{~s}$ at specific annealing primer temperature of $52-62^{\circ} \mathrm{C}, 1 \mathrm{~min}$ at $72^{\circ} \mathrm{C}$, and a final extension of $10 \mathrm{~min}$ at $72^{\circ} \mathrm{C}$. The PCR conditions for exon 9 of PIK3CA were optimized to avoid mispriming with the PIK3CA pseudogene spanning exons 9-13 on chromosome $22 .{ }^{33}$ The PCR products were purified using the exoSAP-IT (USB, Cleveland, OH, USA) and subjected to direct sequencing using $\mathrm{ABI}$ PRISM $^{\mathrm{TM}}$ big Dye terminator v1.1 cycle sequencing Kit (Applied Biosystems, Foster City, CA, USA). Sequencing fragments were detected by capillary electrophoresis using an automated ABI PRISM 310 Genetic Analyzer (Applied Biosystems).

\section{Microsatellite Analysis}

Microsatellite instability status was determined by PCR using fluorochrome-labeled primers for the five recommended microsatellite markers (BAT 25, BAT 26, D2S123, D5S346, and D17S250). ${ }^{34,35}$ Primers and PCR amplification conditions were made as previously described. ${ }^{5}$ Tumors were considered microsatellite instability positive when two or more altered markers were found. Individual PCRs were performed for each marker, and then each sample was capillary electrophoresed on an ABI PRISM 310 Genetic Analyzer (Applied Biosystems).

\section{Statistical Analysis}

Statistical analysis was performed with the statistical package SPSS/win 14.0 (SPSS, Chicago, IL, USA). The following parameters were evaluated: 
age, tumor size, histological type and grade, depth of myometrial invasion, lymphovascular invasion, clinicopathological stage, hormone receptor status, p53 protein expression, microsatellite instability status, presence of PIK3CA, PTEN, CTNNB1, $K-R A S$, and $B-R A F$ mutations, and patients' outcome. A value of $P=0.05$ was considered statistically significant.

\section{Results}

\section{Clinical and Pathologic Findings}

The age of the patients ranged from 35 to 88 years (mean: 66 years). Tumor size varied from 0.4 to $8.8 \mathrm{~cm}$ (mean: $4 \mathrm{~cm}$ ). Of the 109 cases, 102 (94\%) were endometrioid adenocarcinomas and 7 (6\%) were mixed carcinomas ( 5 endometrioid carcinomas (clear cell), and 2 endometrioid carcinomas (serous)). Thirty-four tumors were grade 1, 40 grade 2 , and 35 grade 3 . Most tumors were FIGO stage I (80; $73 \%$ ), 14 stage II, 14 stage III, and 1 stage IV. Myometrial invasion was $\leq 1 / 2$ of the myometrial thickness in 63 cases and $>1 / 2$ in 46 cases. Twentytwo tumors (18 endometrioid adenocarcinomas and
4 mixed carcinomas; 20\%) had lymphovascular invasion. Follow-up information was obtained in 105 of 109 cases (96\%). Ninety-four (90\%) patients were alive without clinical evidence of tumor at a mean follow-up interval of 3.9 years (range: 2 months to 10 years). Tumor persisted or recurred in $3(3 \%)$ patients at a mean follow-up interval of 2.9 years. Two had grade 3 carcinomas (stages IIA and IIIA) and the other had a grade 2 carcinoma (stage IVB). One of the former tumors had lymphovascular invasion. Six $(6 \%)$ patients died of tumor between 10 months and 8.4 years (mean: 3 years) postoperatively. Three had grade 3 carcinomas (stages IB, IC, and IIIA) and the other three patients had grade 2 tumors with lymphovascular invasion (one stage IC and two stage IIIC). Two ( $2 \%$ ) patients died of unrelated causes.

\section{PIK3CA Mutations}

Thirty-five PIK3CA somatic missense mutations were identified in 32 of the 109 (29\%) endometrial carcinomas. Eighteen occurred in the kinase domain (exon 20) and 17 in the helical domain (exon 9). Three carcinomas carried more than one mutation in
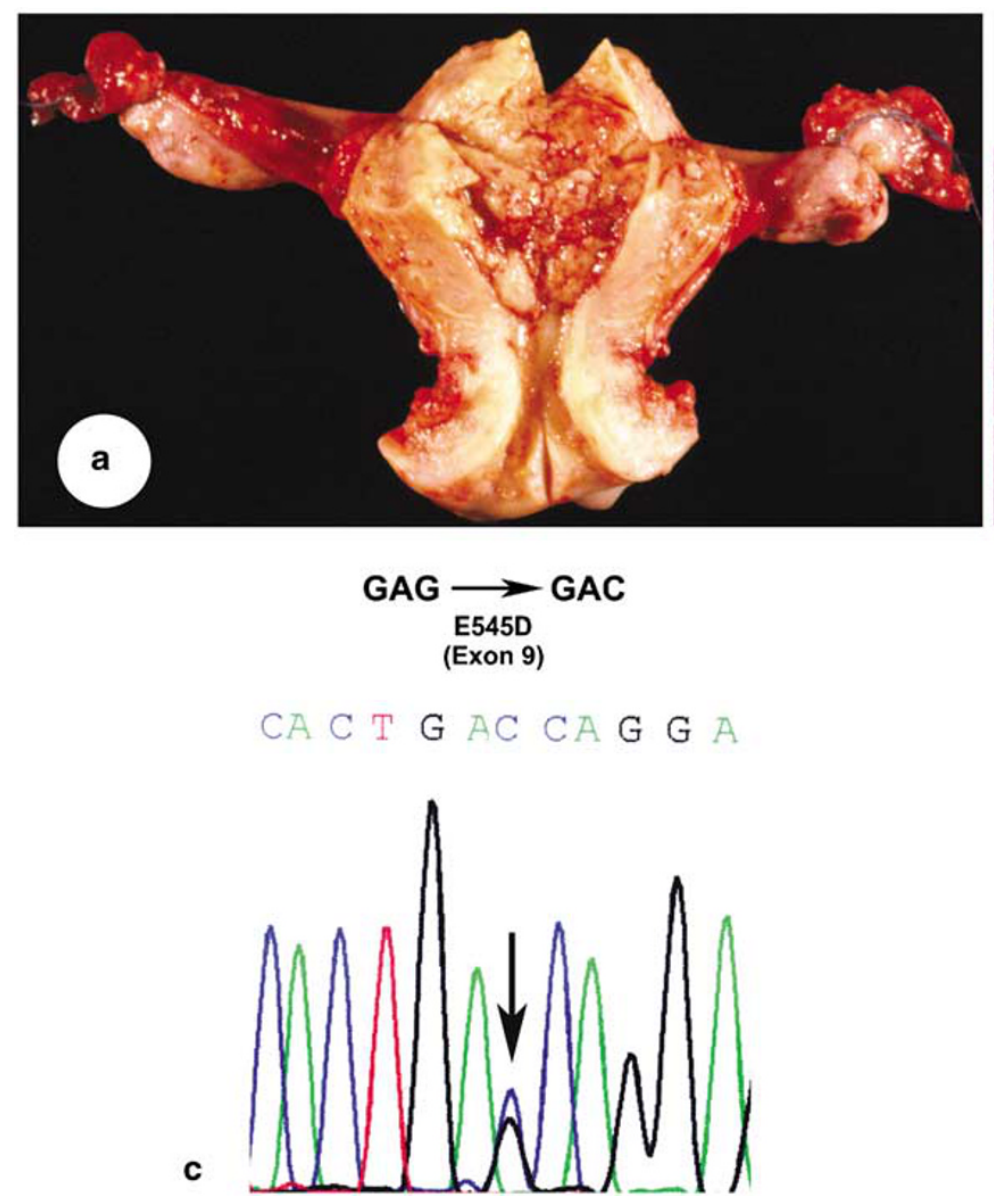
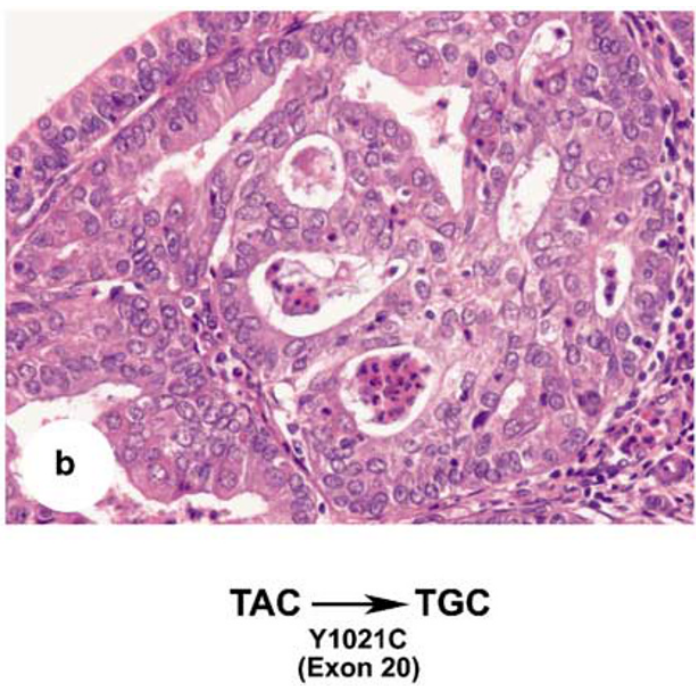

ATT GCAT GCA TT CGA

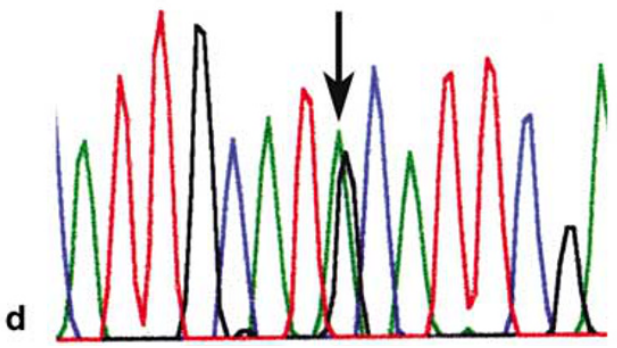

Figure 1 Gross and histological features of an endometrioid carcinoma (a and b) with double mutation in PIK3CA. Sequencing histogram of missense mutations (arrows in $\mathbf{c}$ and $\mathbf{d}$ ) in exon 9 (E545D) and exon 20 (Y1021C). 
Table 1 PIK3CA mutations in endometrial carcinomas

\begin{tabular}{llll}
\hline Exon & Nucleotide & Amino acid & Frequency \\
\hline 9 & A1625T & E542V & 1 \\
9 & G1624A & E542K & 3 \\
9 & G1635C & E545D & 4 \\
9 & A1634G & E545G & 2 \\
9 & G1633A & E545K & 4 \\
9 & A1634C & E545A & 2 \\
9 & A1637G & Q546R & 1 \\
20 & G3052C & D1017H & 1 \\
20 & T3061C & Y1021H & 1 \\
20 & A3062C & Y1021C & 2 \\
20 & A3073G & T1025A & 1 \\
20 & G3103A & A1035T & 1 \\
20 & A3127G & M1043V & 1 \\
20 & A3140G & H1047R & 8 \\
20 & G3155A & T1052K & 1 \\
20 & A3194T & H1065L & 2 \\
\hline
\end{tabular}

${ }^{\mathrm{a}}$ Novel PIK3CA mutation.

exons 9 and 20 (Figure 1). The most commonly mutated codons were 542 (4 cases; $11 \%$ ) and 545 (12 cases; $33 \%$ ) in exon 9 and 1047 ( 8 cases; $22 \%$ ) in exon 20. In addition, a novel mutation was found and confirmed in codon 1017 of exon 20. This mutation, which was a $\mathrm{G}$ to $\mathrm{C}$ transversion converting aspartic to histidine (D1017H), has not been reported previously either in the literature or in the Catalog of Somatic Mutations in Cancer (http:// www.Sanger.ac.uk/genetics/CGP/cosmic). All mutations are listed in Table 1.

\section{Correlations with Clinicopathological Variables}

Almost all (91\%) mutated tumors were pure endometrioid adenocarcinomas, whereas only three of the seven mixed carcinomas (two endometrioid carcinomas (clear cell) and one endometrioid carcinomas (serous)) had PIK3CA mutations. Microdissection was performed in the mixed carcinomas to sample the different components. PIK3CA mutations were found in the endometrioid component in all three cases. All tumors with PIK3CA mutations exhibited myometrial invasion $(P=0.032)$. Although mutated tumors had a higher frequency of lymphovascular invasion (9 of $32 ; 28 \%$ ) than nonmutated carcinomas (13 of $73 ; 18 \%$ ), this association was not statistically significant $(P=0.198)$.

Histological grade varied significantly according to the distribution of PIK3CA mutations between exons 9 and $20(P=0.033)$. Mutations in exon 9 were found more frequently in grade 1 (8 of $14 ; 57 \%$ ) than grade 2 (4 of $14 ; 29 \%$ ) or grade 3 ( 2 of $14 ; 14 \%$ ) tumors. In contrast, mutations in exon 20 were more common in grade $3(9$ of $15 ; 60 \%$ ) than grade 2 (3 of $15 ; 20 \%$ ) or grade 1 (3 of $15 ; 20 \%$ ) carcinomas. Such association was lost when all mutations (exons 9 and 20) were taken into account (Table 2). In addition, both the presence and distribution of
Table 2 Histological grade of endometrial carcinomas according to the presence and distribution of PIK3CA mutations

\begin{tabular}{lccc}
\hline & Grade 1 & Grade 2 & Grade 3 \\
\hline $\begin{array}{l}\text { Endometrial carcinomas } \\
\quad \text { Total }(n=109)\end{array}$ & $34(31 \%)$ & $40(37 \%)$ & $35(32 \%)$ \\
& & & \\
$\begin{array}{l}\text { Endometrial carcinomas } \\
\text { with PIK3CA mutations }\end{array}$ & $12(37 \%)$ & $7(22 \%)$ & $13(41 \%)$ \\
$\quad$ Total $(n=32)$ & & & \\
$\quad$ Exon 9 $(n=14)$ & $8(57 \%)$ & $4(29 \%)$ & $2(14 \%)$ \\
$\quad$ Exon 20 $(n=15)$ & $3(20 \%)$ & $3(20 \%)$ & $9(60 \%)$ \\
$\quad$ Exons 9 and 20 $(n=3)$ & $1(33 \%)$ & $0(0 \%)$ & $2(67 \%)$ \\
$\quad \begin{array}{l}\text { Endometrial carcinomas } \\
\text { without PIK3CA mutations }\end{array}$ & $22(28.5 \%)$ & $33(43 \%)$ & $22(28.5 \%)$ \\
$\quad$ Total $(n=77)$ & & & \\
\hline
\end{tabular}

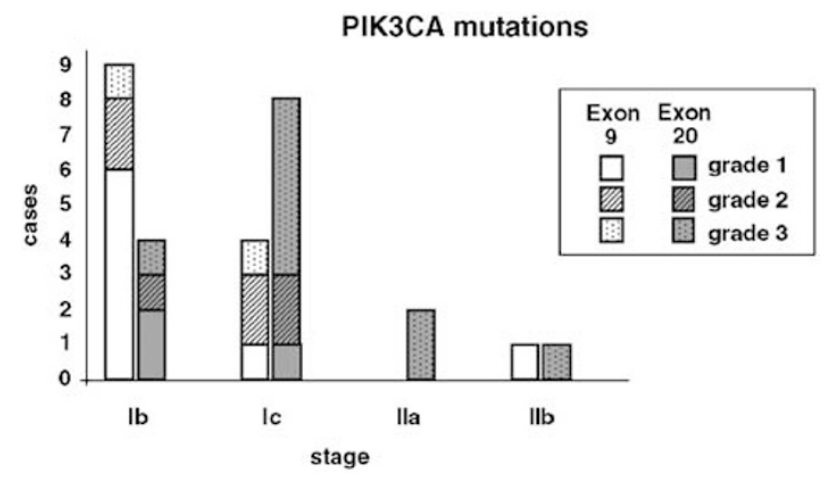

Figure 2 Histological grade and stage of 29 endometrial carcinomas according to the distribution of PIK3CA mutations whether in exon 9 or exon 20.

PIK3CA mutations correlated with stage. None of the tumors confined to the endometrium (stage IA) had PIK3CA mutations. Furthermore, whereas 9 of 14 adenocarcinomas $(64 \%)$ with exon 9 mutations had invaded $\leq 1 / 2$ of the myometrial thickness (stage IB), 11 of $15(73 \%)$ tumors with exon 20 mutations either had deeper myometrial invasion (stage IC) or cervical involvement (stage II) $(P=0.045)$. The three tumors carrying more than one (both exons 9 and 20) mutation exhibited clinicopathological features similar to those of carcinomas with exon 20 mutations only. The distribution of PIK3CA mutations between exons 9 and 20, according to the histological grade and stage of the tumors, is shown in Figure 2. No correlations were found between PIK3CA mutations and other clinicopathological parameters.

\section{Correlations of PIK3CA Mutations with Other Molecular Alterations}

A summary of the molecular genetic findings is shown in Tables 3 and 4. PTEN mutations were 
Table 3 Association of PIK3CA mutations with MI, and PTEN, CTNNB1, K-RAS, and B-RAF mutations

\begin{tabular}{|c|c|c|c|c|c|c|c|c|c|c|}
\hline & \multicolumn{2}{|c|}{ Microsatellite } & \multicolumn{2}{|c|}{ PTEN } & \multicolumn{2}{|c|}{ CTNNB1 } & \multicolumn{2}{|c|}{$K-R A S$} & \multicolumn{2}{|c|}{$B R A F$} \\
\hline & $M I$ & $M S$ & $W T$ & Mut. & Mut. & $W T$ & Mut. & $W T$ & Mut. & $W T$ \\
\hline $\begin{array}{l}\text { PIK3CA } \\
\text { mutation }\end{array}$ & $\begin{array}{l}12 / 37 \\
(32 \%)\end{array}$ & $\begin{array}{l}20 / 72 \\
(28 \%)\end{array}$ & $\begin{array}{l}13 / 50 \\
(26 \%)\end{array}$ & $\begin{array}{l}19 / 59 \\
(32 \%)\end{array}$ & $\begin{array}{c}5 / 24 \\
(21 \%)\end{array}$ & $\begin{array}{l}27 / 85 \\
(32 \%)\end{array}$ & $\begin{array}{c}4 / 19 \\
(21 \%)\end{array}$ & $\begin{array}{l}28 / 90 \\
(31 \%)\end{array}$ & $\begin{array}{c}2 / 2 \\
(100 \%)\end{array}$ & $\begin{array}{c}30 / 107 \\
(28 \%)\end{array}$ \\
\hline
\end{tabular}

MI, microsatellite instability.

Table 4 Molecular genetic findings in endometrial carcinomas with PIK3CA mutations

\begin{tabular}{|c|c|c|c|c|c|c|c|c|c|c|c|c|}
\hline Histology & Grade & Stage & $\begin{array}{l}\text { Vascular } \\
\text { invasion }\end{array}$ & Outcome & $\begin{array}{c}\text { Age } \\
\text { (years) }\end{array}$ & $\begin{array}{c}\text { Tumor } \\
\text { size } \\
(\mathrm{cm})\end{array}$ & $\begin{array}{l}\text { PIK3CA } \\
\text { mut. (exon) }\end{array}$ & MI & PTEN mut. & $\begin{array}{l}\text { CTNNB1 } \\
\text { mut. }\end{array}$ & $\begin{array}{l}\text { K-RAS } \\
\text { mut. }\end{array}$ & $\begin{array}{l}\text { B-RAF } \\
\text { mut. }\end{array}$ \\
\hline EEC & 1 & IB & Yes & DOC & 58 & 2.5 & E545A (9) & No & & & & \\
\hline EEC & 1 & IIB & No & NED & 69 & & E545K (9) & Yes & & $\mathrm{T} 41 \mathrm{~A}$ & G12D & \\
\hline EEC & 2 & IC & No & NED & 77 & 4 & E545D (9) & Yes & 963-968insA & & & \\
\hline EEC & 3 & IC & Yes & NED & 88 & 4.5 & E545G (9) & Yes & G132D & & & \\
\hline EEC & 2 & IB & No & NED & 63 & 6 & Q546R (9) & Yes & 963-968insA & & & \\
\hline EEC & 3 & IB & No & NED & 61 & 4.5 & E542K (9) & No & & & & \\
\hline EEC & 2 & IC & Yes & NED & 82 & 7 & E545K (9) & Yes & 643-645delT & & & \\
\hline EEC & 2 & IB & Yes & NED & 68 & 2 & E545A (9) & No & & & & \\
\hline EEC & 1 & IC & No & NED & 71 & 5 & E545D (9) & No & R130G & & & \\
\hline EEC & 1 & IB & No & NED & 62 & 2 & E545G (9) & Yes & 721-723insT & & & \\
\hline EEC & 1 & IB & No & NED & 64 & 5 & E545D (9) & No & & & G12D & \\
\hline EEC & 1 & IB & No & NED & 73 & 5 & E545K (9) & No & 519-520delCT & S33F & & \\
\hline EEC & 1 & IB & No & NED & 55 & 3 & E542K (9) & Yes & 795-800insA & & & \\
\hline EEC & 1 & IB & No & NED & 69 & 4 & E545K (9) & No & R130Q & & G12A & \\
\hline EEC & 3 & IC & No & NED & 72 & 8.8 & $\begin{array}{l}\text { E542K (9), } \\
\text { D1017H (20) }\end{array}$ & No & 433-437insT & $\mathrm{S} 45 \mathrm{P}$ & & R442T \\
\hline EEC & 3 & IIIA & No & NED & 59 & 3.8 & $\begin{array}{l}\text { E545D (9), } \\
\text { Y1021C (20) }\end{array}$ & No & F81L & & & \\
\hline EEC & 1 & IIA & No & NED & 62 & 3.5 & $\begin{array}{l}\text { E542V (9), } \\
\text { T1052K (20) }\end{array}$ & No & & $\mathrm{S} 45 \mathrm{P}$ & & \\
\hline EEC & 2 & IC & Yes & NED & 61 & 5 & A1035T (20) & No & E7X, R15I & & & \\
\hline EEC & 1 & IC & No & NED & 65 & 5 & H1047R (20) & No & & G34V & & \\
\hline EEC & 3 & IC & No & NED & 58 & 1.5 & H1047R (20) & Yes & K128N & & G12D & \\
\hline EEC & 1 & IB & No & NED & 54 & 1.5 & H1047R (20) & No & S59X, L70F & & & R443W \\
\hline EEC & 3 & IC & Yes & NED & 77 & 4 & H1047R (20) & Yes & 433delTTTTTA & & & \\
\hline EEC & 3 & IIB & No & NED & 69 & 5 & Y1021H (20) & Yes & L146X, 466-469insG & & & \\
\hline Mixed & 3 & IIA & Yes & NED & 64 & 4.2 & H1047R (20) & No & & & & \\
\hline Mixed & 3 & IIA & Yes & AWD & 71 & 7.5 & H1047R (20) & No & & & & \\
\hline EEC & 2 & IB & No & NED & 58 & 4.5 & H1065L (20) & Yes & & & & \\
\hline EEC & 2 & IC & No & NED & 77 & 4.5 & H1065L (20) & No & 993delC & & & \\
\hline EEC & 3 & IC & No & NED & 71 & 8 & M1043V (20) & Yes & 390-391 delA & & & \\
\hline EEC & 1 & IB & No & NED & 66 & 5 & H1047R (20) & No & R173C & & & \\
\hline EEC & 3 & IB & No & NED & 72 & 3.3 & Y1021C (20) & No & & & & \\
\hline EEC & 3 & IC & Yes & NED & 79 & 3.5 & T1025A (20) & No & & & & \\
\hline Mixed & 3 & IC & No & NED & 74 & 6 & H1047R (20) & No & & & & \\
\hline
\end{tabular}

MI, microsatellite instability.

detected in 54\% (59/109), microsatellite instability in $34 \%(37 / 109), \beta$-catenin gene (CTNNB1) mutations in $22 \%$ (24/109), K-RAS mutations in 17\% (19/ $109)$, and $B-R A F$ mutations in $2 \%(2 / 109)$ of cases. PIK3CA mutations coexisted with microsatellite instability, or PTEN, CTNNB1, K-RAS, and B-RAF mutations. Association of PIK3CA and PTEN mutations was found in 19 of $109(17 \%)$ endometrial carcinomas, and PIK3CA mutations were identified more frequently in tumors with PTEN mutations (19 of 59; $32 \%$ ) than in those without PTEN mutations (13 of 50;26\%). Also, PIK3CA mutations coexisted with microsatellite instability in 12 of 109 (11\%) endometrial carcinomas and were more common in tumors with microsatellite instability (12 of 37 ; $32 \%$ ) than in microsatellite stable endometrial carcinomas (20 of $72 ; 28 \%$ ). In contrast, coexistence of PIK3CA and CTNNB1 mutations was found in only 5 of $109(5 \%)$ cases, and the former were less common in endometrial carcinomas with CTNNB 
mutations (5 of $24 ; 21 \%$ ) than in those without CTNNB mutations (27 of $85 ; 32 \%)$. Similarly, a combination of $P I K 3 C A / K-R A S$ mutations occurred in only 4 of 109 (4\%) endometrial carcinomas, and the frequency of $P I K 3 C A$ mutations was lower in endometrial carcinomas with $K-R A S$ mutations (4 of $19 ; 21 \%$ ) than in those without $K-R A S$ mutations (28 of $90 ; 31 \%)$. Nevertheless, the coexistence of PIK3CA mutations with microsatellite instability, PTEN, CTNNB1, or K-RAS mutations did not reach statistical significance. The only two $B-R A F$ mutations detected (2 of 109; $2 \%$ ) were associated with PIK3CA mutations.

\section{Discussion}

The PI3K/AKT pathway is frequently activated in endometrial adenocarcinomas, and a high percentage of mutations of the oncogene PIK3CA have recently been reported. ${ }^{25,27,28}$ However, in contrast to other epithelial cancers, such as colorectal $^{31,36}$ and breast carcinomas, ${ }^{37,38}$ no correlation has been found between the distribution of PIK3CA mutations and grade and stage of disease. Given the recently reported role of PIK3CA mutations in tumor invasion and metastases, ${ }^{29}$ we analyzed the PIK3CA status in a large series of endometrial adenocarcinomas and compared the results, between PIK3CAmutated and PIK3CA-nonmutated groups, with the clinicopathological parameters known to be related to prognosis.

The reported frequency of PIK3CA mutations in endometrial carcinoma ranges from 24 to $39 \% .^{25,27,28}$ Mutations were found predominantly in exon 20 in two studies, ${ }^{25,27}$ and evenly distributed between exons 9 and 20 in a third. ${ }^{28}$ In our series, we encountered 35 mutations in $32(29 \%)$ tumors, and almost all (91\%) were pure endometrioid adenocarcinomas. Microdissection of the different histologic components was performed in the three mixed carcinomas with PIK3CA mutations, which were found in the endometrioid component in all three cases. Eighteen mutations were identified in the kinase domain (exon 20) and 17 in the helical domain (exon 9). Three tumors had more than one mutation in exons 9 and 20. Although, in agreement with previous studies, most mutations (67\%) were clustered in hot spots E542 and E545 of exon 9 and H1047 of exon 20, rare mutations were also found. It was initially thought that rare mutations would not confer as much growth advantage as hot spot mutations; however, recent data indicate that rare PIK3CA mutations in those domains may also result in gain of lipid kinase activity. ${ }^{39}$ Only the helical (exon 9) and kinase (exon 20) domains of PIK3CA have been analyzed in this study. Recently, the frequency and distribution of PIK3CA mutations were reviewed in a total of 2.334 tumor samples corresponding to colon, breast, liver, brain, stomach, lung, and ovary. ${ }^{40}$ It was found that $91 \%$ of the mutations occurred in exons 9 and 20. Moreover, the role of mutations outside of those domains is unclear.

Previous studies in various epithelial cancers have found that PIK3CA mutations are mainly associated with high-grade and invasive tumors. ${ }^{31,36-38}$ For instance, in colorectal carcinomas, their frequency was significantly greater in highgrade and advanced carcinomas $(32 \%)$ than in lowgrade tumors $(3 \%) .{ }^{31}$ Similarly, a higher rate of PIK3CA mutations was found in invasive lobular and ductal breast carcinomas than in other histologic types associated with more favorable prognosis. ${ }^{37}$ Also, it has been shown that PIK3CA mutations occur in colorectal adenomas when the neoplastic cells acquire the ability to invade. ${ }^{31}$ In a comparative study of 44 endometrioid adenocarcinomas and 29 precursor lesions (complex atypical hyperplasias), PIK3CA mutations were identified in $39 \%$ of the former but only $7 \%$ of the latter, suggesting that such mutations represent a late event in endometrial carcinogenesis and are associated with invasion. ${ }^{28}$ However, in contrast to colorectal and breast carcinomas, a correlation between PIK3CA mutations and tumor grade or clinicopathological stage was neither encountered in this ${ }^{28}$ nor in a previous mutational study of 66 endometrial carcinomas. $^{25}$ Conversely, we found that tumor grade varied significantly according to the distribution of PIK3CA mutations between exons 9 and $20(P=0.033)$. Whereas in most grade 1 carcinomas the mutations were identified in exon 9 $(57.1 \%)$, the majority of grade 3 tumors $(60 \%)$ had mutations in exon 20. In addition, all tumors with PIK3CA mutations exhibited myometrial invasion $(P=0.032)$ and, moreover, the depth of myometrial invasion correlated also with the occurrence of the mutations either in exon 9 or exon 20. While most adenocarcinomas with exon 9 mutations (64\%) had invaded only $\leq 1 / 2$ of the myometrial thickness (stage IB), the majority of tumors with exon 20 mutations $(73 \%)$ had either deeper myometrial invasion (stage IC) or cervical involvement (stage II) $(P=0.045)$. Also, mutated tumors had a higher frequency of lymphovascular invasion $(28 \%)$ than nonmutated carcinomas (18\%).

Our findings provide evidence that not only PIK3CA mutations are associated with adverse prognostic parameters in endometrial adenocarcinomas but also their location either in exon 9 or exon 20 carries a different prognosis. The distinct association of different PIK3CA mutations with histologic grade and depth of myometrial invasion is consistent with the recent suggestion that different categories of mutants, as defined by their structural and functional domains, would increase PI3K function by different mechanisms. ${ }^{39}$ Mutations in the kinase domain of exon 20 are close to the hinge region of the catalytic loop and would lead to its activation; in contrast, mutations in the helical domain of exon 9 are clustered on an exposed 
surface patch of the protein and would change its ability to interact with other regulatory proteins, which may vary for each tissue. ${ }^{39}$ Thus, the different mechanisms by which the two categories of PIK3CA mutations induce a gain of function may explain their different impact on tumor grade and myometrial invasion, as found in our study.

Three tumors in our series carried more than one mutation, each in different functional domains of exons 9 and 20. Rare cases of double mutations have also been reported in gastric and breast cancers, ${ }^{23,38}$ but their significance is unknown. Recent investigations suggest that mutations located in the same exon do not produce an additive gain of function, whereas mutations in different exons increase the kinase activity. ${ }^{39}$ Accordingly, the three cases in our study with more than one mutation were advanced tumors and exhibited pathologic features similar to those of carcinomas with mutations in exon 20 only.

We also investigated the occurrence of microsatellite instability and mutations of PTEN, CTNNB1, $K-R A S$, and $B-R A F$ in endometrial carcinomas with PIK3CA mutations. Recent studies in colorectal and breast cancer $^{38,41}$ have claimed that PIK3CA and PTEN mutations are mutually exclusive, suggesting that carcinogenic signaling through this pathway can occur either through activation of PIK3CA or inactivation of PTEN. However, in endometrial carcinoma, three recent studies ${ }^{25,27,28}$ have found the coexistence of PIK3CA and PTEN mutations ranging from 15 to $27 \%$. In one of these investigations, ${ }^{28}$ PTEN mutations occurred in $48 \%$ of complex atypical hyperplasias and $57 \%$ of endometrioid adenocarcinomas; however, the frequency of PIK3CA mutations was significantly lower in hyperplasias than in endometrioid adenocarcinomas (7 vs $39 \%)$. In our series, PTEN mutations were detected in $54 \%$ of endometrioid adenocarcinomas and coexisted with PIK3CA mutations in $17 \%$ of cases. PIK3CA mutations were more common in tumors with PTEN mutations $(32 \%)$ than in those without PTEN mutations (26\%); nevertheless, they were not found to be significantly clustered with PTEN mutations. Microsatellite instability was found in approximately one-third of the tumors in our series; however, association of microsatellite instability with PIK3CA mutations occurred in only $11 \%$ of cases. Although PIK3CA mutations were slightly more frequent in microsatellite instability $(32 \%)$ than in microsatellite stable carcinomas $(28 \%)$, the difference was not statistically significant.

We detected CTNNB1 mutations in $22 \%$ of carcinomas and coexistence with PIK3CA mutations in $5 \%$. We found a lower rate of PIK3CA mutations in tumors with CTNNB1 mutations than in carcinomas without CTNNB1 mutations (21 vs 32\%). Our results are consistent with the finding of CTNNB1 ( $\beta$-catenin gene) mutations predominantly in early-stage tumors associated with favorable prognosis. ${ }^{13,21,42}$
PI3K/AKT and RAS are closely related signaling pathways. RAS can activate PI3K both directly and indirectly. ${ }^{31}$ Mutations in the $K-R A S$ proto-oncogene are identified in approximately $15-30 \%$ of endometrioid adenocarcinomas. ${ }^{9-11}$ In contrast, the frequency of $B-R A F$ mutations is significantly lower. ${ }^{43-45}$ It has been suggested that PIK3CA and $K-R A S$ mutations are mutually exclusive alterations. ${ }^{27}$ However, in our study, K-RAS mutations were detected in $17 \%$ of cases and coexisted with PIK3CA mutations in $4 \%$. This discrepancy might be caused by the difference in the number of cases studied. We found $B-R A F$ mutations in only two cases and, interestingly, they coexisted with PIK3CA mutations, suggesting a possible synergistic effect.

In summary, our study confirms that PIK3CA mutations are frequent in endometrial adenocarcinomas and coexist with microsatellite instability and mutations in PTEN, CTNNB1, K-RAS, and $B-R A F$. It provides evidence that tumors carrying PIK3CA mutations are often high-grade carcinomas associated with myometrial invasion. Moreover, it shows evidence that carcinomas with PIK3CA mutations in exon 20 exhibit higher histological grade and deeper myometrial invasion than those with exon 9 mutations.

\section{Acknowledgement}

This work was supported by Grants FIS PI04-1891, PI06-0950, and RTICCCFIS RD06/0020/0015, Department of Health, Spain, and Marató TV3 2005-47.

\section{Conflict of interest}

None.

\section{References}

1 American Cancer Society. Cancer Facts and Figures 2007. American Cancer Society: Atlanta, GA, 2007.

2 Prat J. Prognostic parameters of endometrial carcinoma. Hum Pathol 2004;35:649-662.

3 Risinger JI, Berchuck A, Kohler MF, et al. Genetic instability of microsatellites in endometrial carcinoma. Cancer Res 1993;53:5100-5153.

4 Caduff RF, Johnston CM, Svoboda-Newman SM, et al. Clinical and pathological significance of microsatellite instability in sporadic endometrial carcinoma. Am J Pathol 1996;148:1671-1678.

5 Catasus L, Machin P, Matias-Guiu X, et al. Microsatellite instability in endometrial carcinomas clinicopathologic correlations in a series of 42 cases. Hum Pathol 1998;29:1160-1164.

6 Tashiro H, Blazes MS, Wu R, et al. Mutations in PTEN are frequent in endometrial carcinoma but rare in other common gynecological malignancies. Cancer Res 1997;57:3935-3940. 
7 Kong D, Suzuki A, Zou TT, et al. PTEN is frequently mutated in primary endometrial carcinomas. Nat Genet 1997;17:143-144.

8 Bussaglia E, del Rio E, Matias-Guiu X, et al. PTEN mutations in endometrial carcinomas: a molecular and clinicopathologic analysis of 38 cases. Hum Pathol 2000;31:312-317.

9 Enomoto $\mathrm{T}$, Inoue $\mathrm{M}$, Perantoni $\mathrm{AO}$, et al. K-Ras activation in premalignant and malignant epithelial lesions of the human uterus. Cancer Res 1991;51: 5308-5314.

10 Caduff RF, Johnston CM, Frank TS. Mutations of the c-Ki-Ras oncogene in carcinoma of the endometrium. Am J Pathol 1995;146:182-188.

11 Lagarda H, Catasus L, Arguelles R, et al. K-ras mutations in endometrial carcinomas with microsatellite instability. J Pathol 2001;193:193-199.

12 Fukuchi T, Sakamoto M, Tsuda H, et al. Beta-catenin mutations in carcinoma of the uterine endometrium. Cancer Res 1998;58:3526-3528.

13 Machin P, Catasus L, Pons C, et al. CTNNB1 mutations and beta-catenin expression in endometrial carcinomas. Hum Pathol 2002;33:206-212.

14 Maxwell GL, Risinger JI, Alvarez AA, et al. Favorable survival associated with microsatellite instability in endometrioid endometrial cancers. Obstet Gynecol 2001;97:417-422.

15 Hirasawa A, Aoki D, Inoue J, et al. Unfavorable prognostic factors associated with high frequency of microsatellite instability and comparative genomic hybridization analysis in endometrial cancer. Clin Cancer Res 2003;9:5675-5682.

16 An HJ, Kim KI, Kim JY, et al. Microsatellite instability in endometrioid type endometrial adenocarcinoma is associated with poor prognostic indicators. Am J Surg Pathol 2007;31:846-853.

17 Risinger JI, Hayes K, Maxwell GL, et al. PTEN mutation in endometrial cancers is associated with favorable clinical and pathologic characteristics. Clin Cancer Res 1998;4:3005-3010.

18 Minaguchi $\mathrm{T}$, Yoshikawa $\mathrm{H}$, Oda $\mathrm{K}$, et al. PTEN mutation located only outside exons 5,6 , and 7 is an independent predictor of favorable survival in endometrial carcinomas. Clin Cancer Res 2001;7: 2636-2642.

19 Salvesen HB, MacDonald N, Ryan A, et al. PTEN methylation is associated with advanced stage and microsatellite instability in endometrial carcinoma. Int J Cancer 2001;91:22-26.

20 Saegusa $\mathrm{M}$, Hashimura $\mathrm{M}$, Yoshida $\mathrm{T}$, et al. Beta-catenin mutations and aberrant nuclear expression during endometrial tumorigenesis. Br J Cancer 2001;84:209-217.

21 Brachtel E, Sanchez C, Moreno G, et al. Distinct molecular alterations in complex endometrial hyperplasia (CEH) with and without immature squamous metaplasia (squamous morules). Am J Surg Pathol 2005;29:1322-1329.

22 Campbell IG, Russell SE, Choong DY, et al. Mutation of the PIK3CA gene in ovarian and breast cancer. Cancer Res 2004;64:7678-7681.

23 Lee JW, Soung YH, Kim SY, et al. PIK3CA gene is frequently mutated in breast carcinomas and hepatocellular carcinomas. Oncogene 2005;24: 1477-1480.

24 Samuels Y, Velculescu VE. Oncogenic of PIK3CA in human cancers. Cell Cycle 2004;3:1221-1224.
25 Oda K, Stokoe D, Taketani Y, et al. High frequency of coexistent mutations of PIK3CA and PTEN genes in endometrial carcinoma. Cancer Res 2005;65: 10669-10673

26 Stambolic V, Suzuki A, de la Pompa JL, et al. Negative regulation of $\mathrm{PKB} / \mathrm{AKT}$ dependent cell survival by the tumor suppressor PTEN. Cell 1998;95:29-39.

27 Velasco A, Bussaglia E, Pallares J, et al. PIK3CA gene mutations in endometrial carcinoma. Correlation with PTEN and K-Ras alterations. Hum Pathol 2006;37: 1465-1472.

28 Hayes MP, Wang H, Espinal-Witter R, et al. PIK3CA and PTEN mutations in uterine endometrioid carcinoma and complex atypical hyperplasia. Clin Cancer Res 2006;12:5932-5935.

29 Samuels Y, Diaz Jr LA, Schmidt-Kittler O, et al. Mutant PIK3CA promotes cell growth and invasion of human cancer cells. Cancer Cell 2005;7:561-573.

30 Silverberg SG, Kurman RJ, Nogales F, et al. Tumours of the uterine corpus. In: Tavassoli FA, Devilee P (eds). World Health Organization Classification of Tumours. Pathology and Genetics of Tumours of the Breast and Female Genital Organs. IARC Press: Lyon, 2003, pp 217-257.

31 Samuels Y, Wang Z, Bardelli A, et al. High frequency of mutations of the PIK3CA gene in human cancers. Science 2004;304:554.

32 Feng YZ, Shiozawa T, Miyamoto $\mathrm{T}$, et al. BRAF mutation in endometrial carcinoma and hyperplasia: correlation with KRAS and p53 mutations and mismatch repair protein expression. Clin Cancer Res 2005;11:6133-6138.

33 Müller C, Miller CW, Hofmannn W-K, et al. Rare mutations of the PIK3CA gene in malignancies of the hematopoietic system as well as endometrium, ovary, prostate and osteosarcomas, and discovery of a PIK3CA pseudogene. Leuk Res 2007;31:27-32.

34 Bocker T, Diermann J, Friedl W, et al. Microsatellite instability analysis: a multicenter study for reliability and quality control. Cancer Res 1997;57:4739-4743.

35 Boland CR, Thibodeau SN, Hamilton SR, et al. A National Cancer Institute workshop on microsatellite instability for cancer detection and familial predisposition: development of international criteria for the determination of microsatellite instability in colorectal cancer. Cancer Res 1998;58:5248-5257.

36 Samuels Y, Ericson K. Oncogenic PI3K and its role in cancer. Curr Opin Oncol 2006;18:77-82.

37 Buttitta F, Felicioni L, Barassi F, et al. PIK3CA mutation and histological type in breast carcinoma: high frequency of mutations in lobular carcinoma. J Pathol 2006;208:350-355.

38 Saal LH, Holm K, Maurer M, et al. PIK3CA mutations correlate with hormone receptors, node metastasis, and ERBB2, and are mutually exclusive with PTEN loss in human breast carcinoma. Cancer Res 2005;65:2554-2559.

39 Gymnopoulos M, Elsliger M-A, Vogt PK. Rare cancerspecific mutations in PIK3CA show gain of function. Proc Natl Acad Sci 2007;104:5569-5574.

40 Bader AG, Kang S, Zhao L, et al. Oncogenic PI3K deregulates transcription and translation. Nat Rev 2005;5:921-929.

41 Frattini M, Signoroni S, Pilotti S, et al. Phosphatase protein homologue to tensin expression and phosphatidylinositol-3 phosphate kinase mutations in colorectal cancer. Cancer Res 2005;65:11227. 
42 Palacios J, Catasus L, Moreno-Bueno G, et al. Beta- and gamma-catenin expression in endometrial carcinoma. Relationship with clinicopathological features and microsatellite instability. Virchows Arch 2001;438: 464-469.

43 Mutch DG, Powell MA, Mallon MA, et al. RAS/RAF mutation and defective DNA mismatch repair in endometrial cancers. Am J Obstet Gynecol 2004;190:935-942.
44 Salvesen HB, Kumar R, Stefansson I, et al. Low frequency of BRAF and CDKN2A mutations in endometrial cancer. Int J Cancer 2005;115: 930-934.

45 Pappa KI, Choleza M, Markaki S, et al. Consistent absence of BRAF mutations in cervical and endometrial cancer despite K-Ras mutation status. Gynecol Oncol 2006;100:596-600. 